\title{
Auswirkungen des Arbeitsgesetzes auf die ärztliche Weiterbildung und deren zeitlicher Aufwand aus Sicht der Leiterinnen und Leiter von Weiterbildungsstätten
}

Im Frühjahr 2006 wurden die Leiterinnen und Leiter von 1370 anerkannten Weiterbildungsstätten zu den Resultaten der Assistentenumfrage 2005, zu den Auswirkungen des Arbeitsgesetzes auf die Weiterbildung und die Patientenversorgung sowie ihrem eigenen zeitlichen Aufwand für die Weiterbildung befragt. Während die Assistenzärzte keine Beeinflussung von Weiterbildung und Patientenversorgung empfinden, sehen die Leiter einen negativen Einfluss in beiden Gebieten. Die Hälfte der Leiterinnen und Leiter wendet mehr als sechs Stunden pro Woche für die Weiterbildung der Assistenzärztinnen und -ärzte auf. Diese Zahl drückt den Stellenwert aus, den die Leiterinnen und Leiter der ärztlichen Weiterbildung beimessen; der geschätzte Zeitaufwand erlaubt aber keinen Rückschluss auf deren Kosten oder Effizienz.

Michael Siegrist ${ }^{a}$,

Pascale Orlow ${ }^{a}$, Max Giger ${ }^{b}$

a Sozialforschungsstelle der Universität Zürich

b Mitglied des Zentralvorstandes der FMH, Ressort Medical Education
Korrespondenz:

Dr. med. Max Giger

FMH

Elfenstrasse 18

CH-3000 Bern 15

max.giger@hin.ch
Um die Weiterbildungssituation zuverlässig beschreiben zu können, müssen neben den Assistenzärztinnen und -ärzten [1] auch die Leiterinnen und Leiter von Weiterbildungsstätten regelmässig befragt werden. Die im Jahr 2004 durchgeführte Umfrage bei den Leiterinnen und Leitern von Weiterbildungsstätten zeigte, dass die Ergebnisse der Assistentenumfrage in erfreulichem Umfang Massnahmen zur Qualitätssteigerung der Weiterbildung auslösten [2]. Weiter zeigte die Befragung, dass eine Mehrheit der Leiter die Assistenten als weniger einsatzfreudig wahrnimmt im Vergleich zu früher.

Mit der Umfrage 2006 bei den Leiterinnen und Leitern wollten wir überprüfen, ob diese die Umfrage bei den Assistenzärztinnen und -ärzten immer noch überwiegend positiv einschätzen und durch deren Resultate Massnahmen zur Verbesserung der Weiterbildung einleiten. Die Umfrage 2005 bei den Assistenzärzten zeigte, dass für eine Mehrheit der Assistenzärzte das Arbeitsgesetz keinen negativen Einfluss auf die Weiterbildung und die Arbeit zu haben scheint [1]. In der vorliegenden Untersuchung wurde überprüft, wie die Leiter den Einfluss des Arbeitsgesetzes auf die Weiterbildung und die Arbeit der
Assistenzärzte im Vergleich zur Einschätzung der Assistenzärzte beurteilen. Abschliessend wurde erhoben, wie viele Stunden die Leiter durchschnittlich pro Monat für die Weiterbildung von Assistenzärzten aufwenden.

\section{Methode}

\section{Stichprobe}

Im Rahmen der statistischen Erhebung bei den Leitern und Leiterinnen der Weiterbildungsstätten wurden Fragen $\mathrm{zu}$ unterschiedlichen Aspekten der Weiterbildung gestellt. Die Umfrage erfolgte im Frühling 2006 auf elektronischem Weg bei 1370 von der FMH anerkannten Weiterbildungsstätten. Die Frage mit der tiefsten Antwortquote wurde von $62 \%$ der Leiterinnen und Leiter beantwortet. Die Leiterinnen und Leiter wurden nach ihrer Funktion innerhalb der Institution befragt. Von den Teilnehmenden der Befragung gaben 80,3\% die Funktionen «Chefarzt», 14,5\% «Leitender Arzt», 0,9\% «Oberarzt» und $4,3 \%$ «anderes» an. Die Ergebnisse dieser Umfrage zum Arbeitsgesetz wurden mit denjenigen der Assistenzärzte vom Spätsommer 2005 verglichen. 
Für die Berechnung der Prozentwerte wurde als Basis die Zahl der Personen berücksichtigt, die die entsprechenden Fragen beantwortet hatten.

\section{Fragebogen}

Die Befragten wurden in erster Line mit Aussagen konfrontiert. Für diese Aussagen standen sechs Antwortkategorien zur Auswahl, von 1 «trifft überhaupt nicht zu» bis 6 «trifft voll und ganz zu». Die Zahlen dazwischen waren nicht verbal verankert. Der Fragebogen beinhaltete drei Themenkreise neben den jährlichen Erhebungen zur Struktur der Weiterbildungsstätten. Zuerst wurden Fragen zum Bericht über die Weiterbildungsqualität aus der Sicht der Assistenzärzte 2005 gestellt, danach Fragen zur Weiterbildungs- und Arbeitssituation bei den Assistenzärzten und zum Schluss Fragen zum persönlichen

\section{Abbildung 1}

Weiterbildung: Antworten auf das Statement «Die vom Gesetz vorgeschriebene Arbeitszeitregelung wirkt sich negativ auf die Weiterbildung der Assistenzärzte aus».

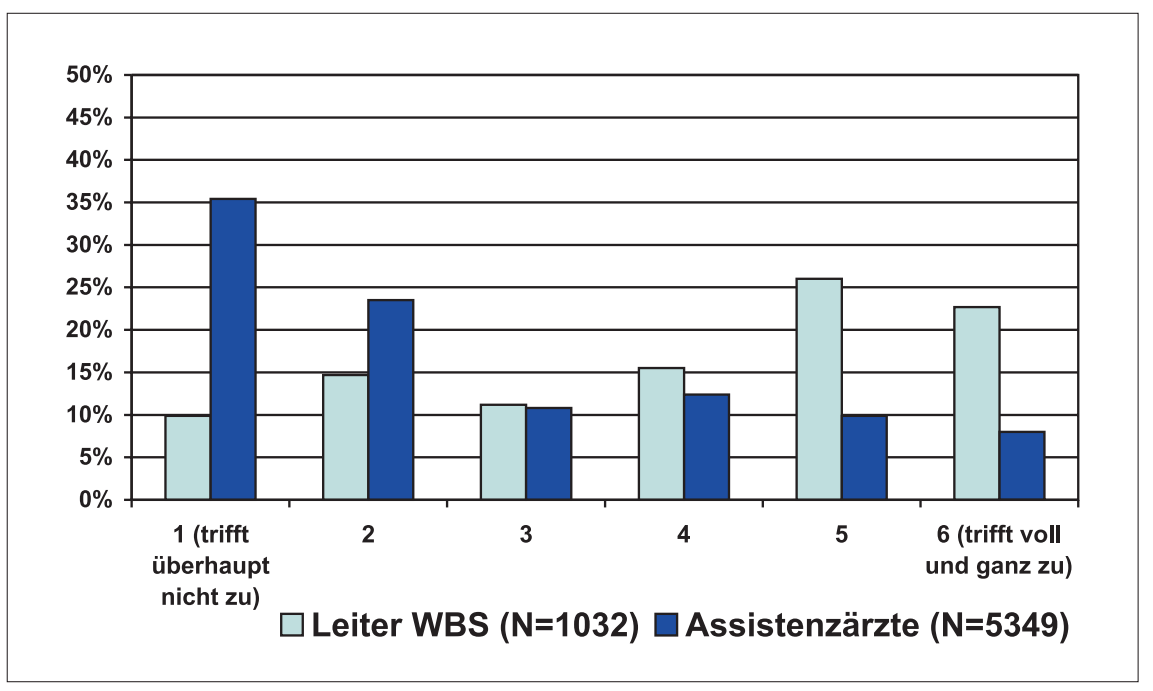

Abbildung 2

Patientenversorgung: Antworten auf das Statement «Die vom Gesetz vorgeschriebene Arbeitszeitregelung wirkt sich negativ auf die Arbeit der Assistenzärzte am Patienten aus».

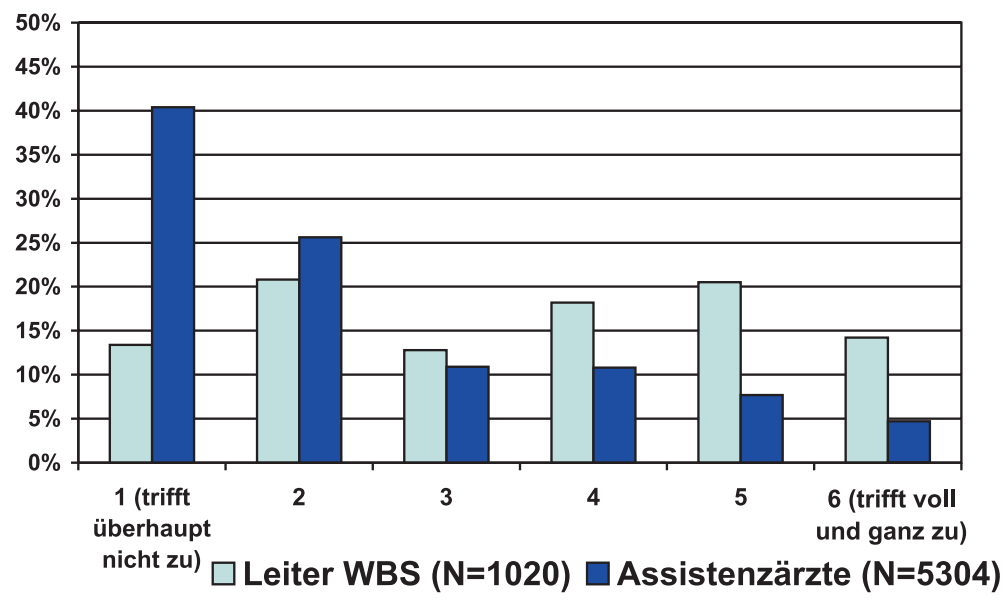

zeitlichen Aufwand für die Weiterbildung der Assistenzärzte. Leitern von 1000 Weiterbildungsstätten, die einen Bericht erhalten hatten, wurden spezifische Fragen dazu gestellt. Die Befragten konnten angeben, welche Aktivitäten durch den Bericht ausgelöst wurden. Die genauen Fragestellungen sind im Anhang aufgeführt.

Die Leiterinnen und Leiter wurden gefragt, wie viele Stunden sie durchschnittlich pro Monat im Rahmen der Weiterbildung von Assistenzärzten für verschiedene Tätigkeiten aufwenden. Die folgenden sechs Bereiche wurden dabei berücksichtigt: Feedbackgespräche zur Weiterbildung von Assistenzärzten, Kadergespräche zur Beurteilung der Assistenzärzte, persönliche Vorbereitung für Weiterbildung von Assistenzärzten, Vorträge für Assistenzärzte, Kurse für Assistenzärzte sowie Instruktionen und Anleitungen am Patienten (Bedsideteaching/Operationsassistenz).

\section{Ergebnisse}

Beurteilung des Berichts über die Weiterbildung aus der Sicht der Assistenzärzte 2005 Bei $77 \%{ }^{*}(\mathrm{~N}=720)$ der Weiterbildungsstätten wurden die Ergebnisse mit Oberärzten/leitenden Ärzten besprochen. Bei $67 \%(\mathrm{~N}=620)$ Weiterbildungsstätten wurden die Ergebnisse mit Assistenzärzten besprochen, und bei $60 \%(\mathrm{~N}=554)$ der Weiterbildungsstätten wurden konkrete Schritte für die Verbesserung der Weiterbildungsqualität eingeleitet. Für $65 \%(\mathrm{~N}=554)$ der Leiterinnen und Leiter von Weiterbildungsstätten waren die Resultate Anlass, die Weiterbildung zu überdenken.

\section{Arbeitsgesetz}

Abbildung 1 zeigt die Antworten auf die Frage, ob sich die gesetzlich vorgeschriebene Arbeitszeitregelung negativ auf die Weiterbildung der Assistenzärzte auswirkt. Die Abbildung zeigt, dass die Situation von den Leitern der Weiterbildungsstätten deutlich anders eingeschätzt wird als von den Assistenzärzten: Über 50\% der Leiterinnen und Leiter von Weiterbildungsstätten sind der Meinung, dass die reduzierte Arbeitszeit sich negativ auf die Weiterbildung auswirkt; hingegen ist eine Mehrheit der Assistenzärzte der Ansicht, dass sich die Arbeitszeitregelung nicht negativ auf ihre Weiterbildung auswirkt.

Ein ähnliches Muster ergab sich für die Auswirkungen der Arbeitszeitregelung auf die Arbeit der Assistenzärzte am Patienten (Abb. 2). Eine Mehrheit der Assistenzärzte kann keine negativen Auswirkungen feststellen. Bei den Leitern und Leiterinnen von Weiterbildungsstätten über- 
wiegen dagegen jene, welche einen negativen Einfluss auf die Arbeit der Assistenzärzte am Patienten erkennen.

\section{Zeitlicher Aufwand der Leiterinnen und Leiter von WBS für die Weiterbildung}

Eine explorative Datenanalyse zeigte, dass es Ausreisser (extrem hohe Werte) gab. Aus diesem Grunde werden im folgenden nur robuste Kennwerte wie der Median und der Interquartilrange angegeben. Beides sind Kennwerte, die durch Ausreisser kaum beeinflusst werden. Die angegebenen Stunden für die sechs Teilbereiche der Weiterbildung wurden aufsummiert. Der Median für alle Fachrichtungen $(\mathrm{N}=806)$ beträgt 24,0 Stunden/Monat $(\mathrm{IQR}=24,3)$.

In Tabelle 1 ist der geschätzte Aufwand für die Weiterbildung der Leiterinnen und Leiter für die sieben Fachrichtungen mit den meisten Assistenzärzten aufgeführt. Der Median der einzelnen Fachrichtungen schwankt zwischen 20 und 32 Stunden pro Monat.

\section{Diskussion}

Ein grosser Teil der Leiterinnen und Leiter von Weiterbildungsstätten gibt an, aufgrund der Ergebnisse der Assistentenumfrage die Weiterbildung hinterfragt oder gar Veränderungsprozesse eingeleitet zu haben. Dies ist ein sehr erfreuliches Ergebnis. Es zeigt auch, dass mit der Umfrage bei den Assistenzärzten das Ziel erreicht wird, den Leitern und Leiterinnen möglichst konkrete Hinweise auf Veränderungspotential zu geben.

Die Auswirkungen des Arbeitsgesetzes werden von den Assistenzärzten ganz anders beurteilt als von den Leiterinnen und Leitern. Für die meisten Assistenzärzte hat das Arbeitsgesetz keine negativen Auswirkungen auf die Weiterbildung oder auch auf die Arbeit am Patienten. Die Lei-

\section{Tabelle 1}

Median und IQR der selbstgeschätzten Zahl der Stunden pro Monat, die die Leiterinnen und Leiter für die Weiterbildung aufwenden; aufgelistet nach den sieben Fachrichtungen mit den meisten Assistenzärzten.

\begin{tabular}{lllr} 
Fachrichtung & Median (Std./Monat) & IQR & N \\
\hline Anästhesiologie & 32,0 & 35,9 & 34 \\
\hline $\begin{array}{l}\text { Orthopädische Chirurgie und Traumatologie } \\
\text { des Bewegungsapparates }\end{array}$ & 28,1 & 30,0 & 33 \\
\hline Chirurgie & 27,5 & 23,2 & 75 \\
\hline Kinder- und Jugendmedizin & 27,0 & 23,6 & 27 \\
\hline Innere Medizin & 27,0 & 29,5 & 125 \\
\hline Gynäkologie und Geburtshilfe & 22,3 & 23,6 & 50 \\
\hline Psychiatrie und Psychotherapie & 20,0 & 18,6 & 71
\end{tabular}

terinnen und Leiter können demgegenüber in beiden Bereichen negative Auswirkungen des Arbeitsgesetzes erkennen. Eine mögliche Erklärung dieser unterschiedlichen Einschätzung könnte darin liegen, dass der Erfahrungshintergrund der beiden Gruppen vollständig unterschiedlich ist und deshalb auch ein unterschiedlicher Vergleichsmassstab benutzt wird.

Die Umfrage bei den Leiterinnen und Leitern zeigt, dass sich die meisten Leiterinnen und Leiter auch zeitlich stark um die Weiterbildung kümmern. Fünfzig Prozent der Befragten geben an, mehr als 24 Stunden pro Monat für die Weiterbildung aufzuwenden. Die Hälfte der Leiterinnen und Leiter der sieben Fachrichtungen mit den meisten Assistenzärzten wenden zwischen 5 und 8 Stunden pro Woche für die ärztliche Weiterbildung auf (Abb. 3). Diese Werte liegen im Bereich, wie sie 2000 und 2002 an den Universitätsspitälern Lausanne und Zürich erhoben wurden $[3,4]$.

Bei der Interpretation der Zeitangaben muss allerdings beachtet werden, dass es sich zum einen um retrospektive Schätzungen handelt und zum andern um Selbsteinschätzungen. Auch können die Fragen unterschiedlich interpretiert worden sein. Diese Nachteile könnten nur dann ausgeschaltet werden, wenn nach einer detaillierten Definition der für die Weiterbildung relevanten Anteile die Tätigkeiten der Leiter genauestens protokolliert würden. Dies wäre mit einem sehr grossen finanziellen Aufwand verbunden. Weiter handelt es sich bei den Zahlen lediglich um den Aufwand der Leiterinnen und Leiter. Würden zusätzlich die Aufwendungen der leitenden Ärzte, Oberärzte und des paramedizinischen Personals berücksichtigt, so würde sich daraus eine entsprechend höhere Zahl von Stunden für die Weiterbildung ergeben. Diese Zahlen müssten den Auswirkungen auf die Leistungen der Assistenzärzte gegenübergestellt werden, um über die Effektivität und Effizienz der Weiterbildung eine zuverlässige Aussage machen zu können.

\section{Literatur}

1 Siegrist M, Orlow P, Giger M. Weiterbildung aus der Sicht der Assistenzärzte: Die wichtigsten Resultate der Umfrage 2004 bei Assistenzärztinnen und -ärzten über die Weiterbildung. Schweiz Ärztezeitung 2005;86(7):412-23.

2 Siegrist M, Giger M. Assistenten- und Assistentinnenumfrage 2003 aus Sicht der Leiterinnen und Leiter von Weiterbildungsstätten. Schweiz Ärztezeitung 2004;85(42):2262-6.

3 Schenker L. Persönliche Mitteilung.

4 Vetter W. Persönliche Mitteilung. 


\section{Abbildung 3}

Selbstgeschätzte Stunden pro Woche, die die Leiterinnen und Leiter für die Weiterbildung aufwenden; aufgelistet nach den sieben Fachrichtungen mit den meisten Assistenzärzten. Die Antworten von den Befragten mit 20 Std./Woche oder mehr wurden nicht berücksichtigt.

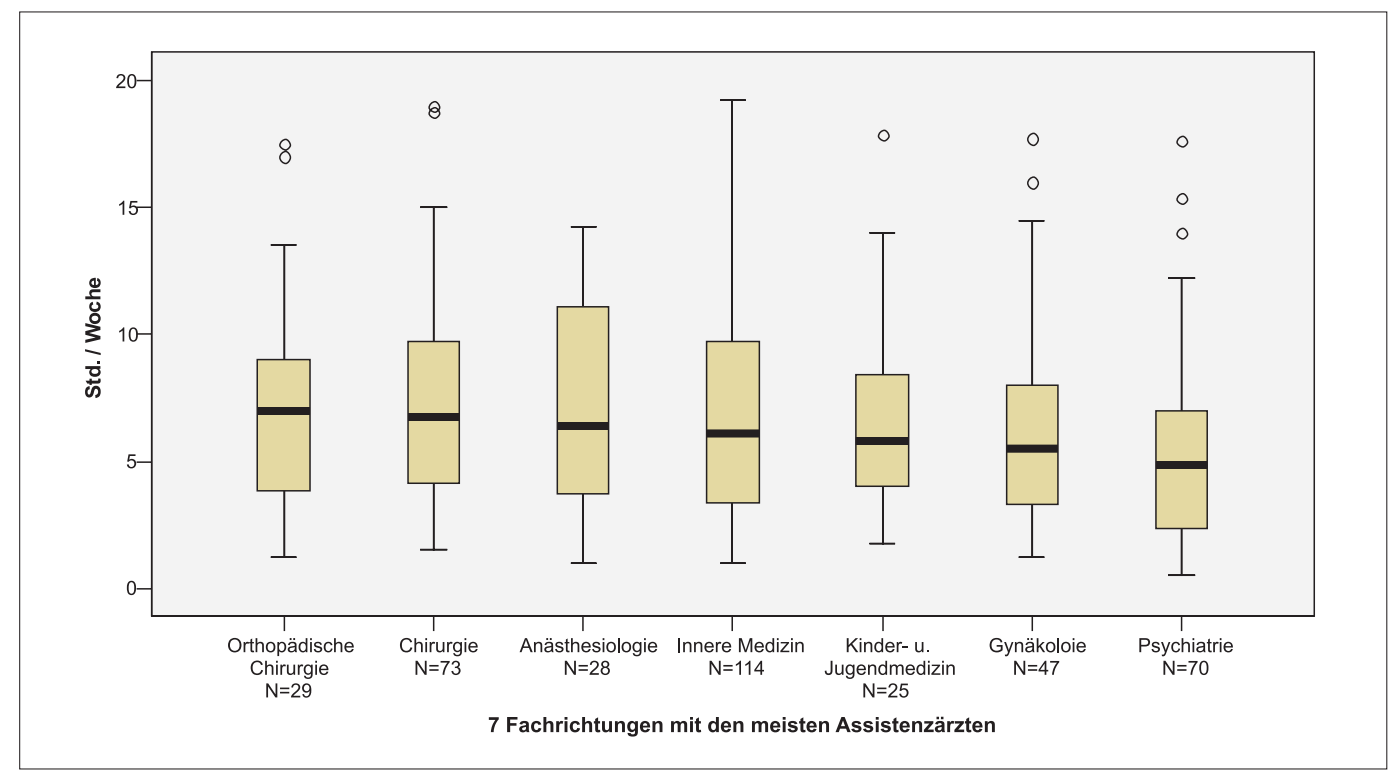

\section{Anhang}

\section{Fragebogen der Umfrage 2006 bei den Leiterinnen und Leitern}

Die folgenden Fragen wurden von den Leitern und Leiterinnen von Weiterbildungsstätten beantwortet.

\section{Frage}

1. Welche Funktion haben Sie inne?

2. Haben Sie einen Bericht zur Umfrage 2005 erhalten, in dem die Resultate für Ihre WBS aufgeführt sind?

3. Welche der folgenden Aktivitäten wurden durch den Bericht ausgelöst? (Mehrfachnennungen sind möglich)

4. Durch den Bericht habe ich etwas Neues über unsere Weiterbildungsstätte erfahren.

5. Unsere Weiterbildungsstätte wurde in der Umfrage gerecht beurteilt.

6. Die Resultate der Umfrage waren Anlass für mich, unsere Weiterbildung zu überdenken.

7. Die Resultate der Umfrage 2005 sind wegen ungenügender Beteiligung der Assistenten für unsere Weiterbildungsstätte statistisch nicht aussagekräftig.

8. In der vertraglich geregelten Arbeitszeit können die Assistenzärzte die Arbeit zu meiner Zufriedenheit erfüllen

9. Die vom Gesetz vorgeschriebene Arbeitszeitregelung wirkt sich negativ auf die Weiterbildung der Assistenzärzte aus.

10. Die vom Gesetz vorgeschriebene Arbeitszeitregelung wirkt sich negativ auf die Arbeit der Assistenzärzte am Patienten aus.

11. Wie oft können Ihre Assistenzärzte die Ruhezeiten nicht gemäss dem Arbeitsgesetz einhalten?

12. Die im Arbeitsvertrag vereinbarte Arbeitszeit (100\%-Pensum $=$ maximal 50 Std./Wo) bei den Assistenzärzten wird umgesetzt.

13. Wie viele Stunden wenden Sie durchschnittlich pro Monat im Rahmen der Weiterbildung von Assistenzärzten für die folgenden Tätigkeiten auf?

\section{Antwortkategorien}

Chefarzt; leitender Arzt; Oberarzt; anderes

Ja; nein

Bericht noch nicht gelesen; Bericht gelesen; Ergebnisse mit Oberärzten/ leitenden Ärzten besprochen; Ergebnisse mit Assistenzärzten besprochen; Konkrete Schritte für die Verbesserung der Weiterbildungsqualität eingeleitet; Schreiben/Kritik/Nachfragen an $\mathrm{FMH} /$ Sozialforschungsstelle Uni ZH

1 trifft überhaupt nicht zu; $2 ; 3 ; 4 ; 5 ; 6$ trifft voll und ganz zu

1 trifft überhaupt nicht zu; $2 ; 3 ; 4 ; 5 ; 6$ trifft voll und ganz zu

1 trifft überhaupt nicht zu; $2 ; 3 ; 4 ; 5 ; 6$ trifft voll und ganz zu

1 trifft überhaupt nicht zu; $2 ; 3 ; 4 ; 5 ; 6$ trifft voll und ganz zu

1 trifft überhaupt nicht zu; $2 ; 3 ; 4 ; 5 ; 6$ trifft voll und ganz zu

1 trifft überhaupt nicht zu; $2 ; 3 ; 4 ; 5 ; 6$ trifft voll und ganz zu

1 trifft überhaupt nicht zu; $2 ; 3 ; 4 ; 5 ; 6$ trifft voll und ganz zu

Nie; sehr selten; gelegentlich; regelmässig

Ja; nein

Gespräche zur Karriereplanung von Assistenzärzten: $h /$ Monat; Feedbackgespräche zur Weiterbildung von Assistenzärzten: h/Monat; Kadergespräche zur Beurteilung der Assistenzärzte: $h$ /Monat; Persönliche Vorbereitung für Weiterbildung von Assistenzärzten: $h /$ Monat; Vorträge für Assistenzärzte: h/Monat; Kurse für Assistenzärzte: h/Monat; Instruktionen und Anleitungen am Patienten (Bedsideteaching/Operationsassistenz): h/Monat 